DOI: https://doi.org/10.11144/Javeriana.upsy18-5.ccpr

\title{
Conciencia de las capacidades personales y rendimiento académico en la implementación de un programa psicoeducativo*
}

\section{Awareness of Personal Capacities and Academic Performance in the Implementation of a Psychoeducational Program}

Recepción: 06 Julio 2019 | Aceptación: 02 Noviembre 2019

\author{
EsPeranza MÁrquez López \\ Universidad de Sevilla, España \\ ORCID: http://orcid.org/0000-0002-5697-5320 \\ Daniel Holgado Ramos \\ Universidad de Sevilla, España \\ ORCID: http://orcid.org/0000-0001-6350-7982 \\ IsIDRO MAYA JARIEGO ${ }^{a}$ \\ Universidad de Sevilla, España \\ ORCID: http://orcid.org/0000-0002-2568-5048
}

a Autor de correspondencia. Correo electrónico: isidromj@us.es

Para citar este artículo: Márquez López, E., Holgado Ramos, D., Maya Jariego, I. (2019). Conciencia de las capacidades personales y rendimiento académico en la implementación de un programa psicoeducativo. Universitas Psychologica, 18(5), 1-18. https://doi.org/1 0.11144/Javeriana.upsy18-5.ccpr

\section{RESUMEN}

La mejora de la experiencia educativa de los menores y el desarrollo de competencias personales reducen el riesgo de trabajo infantil. En este estudio valoramos el impacto de una intervención psicoeducativa para la prevención del trabajo infantil en dos barrios de la periferia de Lima. Con un diseño pretest-postest, evaluamos los cambios en la autoestima, la autoeficacia, las habilidades sociales, la asistencia a la escuela y el rendimiento académico de 48 alumnos de entre 8 y 13 años $(M=10.38, D E=1.22)$. Los resultados mostraron una mejora en la autoestima percibida y un descenso en la autoeficacia social y el auto-informe de habilidades sociales. La evaluación cualitativa de la percepción de los cambios por parte de los estudiantes, los padres y los docentes mostró mejoras en el desarrollo socio-afectivo de los participantes. La evaluación del programa sugiere la existencia de un proceso de toma de conciencia de las capacidades personales por parte de los participantes, junto con pequeñas mejoras en el ámbito académico que pueden repercutir indirectamente en el riesgo del trabajo infantil.

Palabras clave

Edúcame Primero Perú; competencias personales; auto regulación; habilidades sociales; prevención; trabajo infantil.

\section{ABSTRACT}

The improvement of the educational experience of minors and the development of personal skills reduce the risk of child labor. In this study we assess the impact of a psychoeducational intervention for the prevention of child labor in two neighborhoods in the periphery of Lima. With a pretest-posttest design, we evaluate the changes in self-esteem, self-efficacy, social skills, school attendance and academic performance of 48 students between 8 and 13 years old $(M=10.38, S D=1.22)$. The results 
showed an improvement in perceived self-esteem and a decrease in social self-efficacy and social skills selfreport. The qualitative evaluation of the perception of changes by students, parents and teachers demonstrated improvements in the socio-affective development of the participants. The evaluation of the program implies the existence of a process of awareness of the personal abilities of the participants, together with small improvements in the academic field that can have an indirect impact on the risk of child labor.

Keywords

Edúcame Primero Perú; personal skills; self-regulation; social skills; prevention; child labor.

La Organización Internacional del Trabajo (OIT) considera que el trabajo infantil es toda aquella actividad laboral "que priva a los niños de su niñez, su potencial y su dignidad, y que es perjudicial para su desarrollo físico y psicológico" (OIT, 2004, p. 16). Para afrontarlo, propone programas dirigidos a promover la educación de calidad, junto con la concienciación del contexto familiar y comunitario de los menores, desde la primera infancia (OIT, 2017a).

En este contexto, se han desarrollado programas de intervención psicosocial diseñados para prevenir el trabajo infantil, disminuir su prevalencia y proporcionar herramientas y estrategias de desarrollo a los menores que viven en contextos de riesgo. En este estudio se analiza el impacto de una intervención psicoeducativa, "Edúcame Primero Perú", que pretende fortalecer las competencias psicosociales en los menores y facilitar un contexto familiar e institucional que contribuya a su desarrollo personal.

\section{Causas del trabajo infantil}

El trabajo infantil es un fenómeno multicausal que afecta al desarrollo psicosocial de los niños y niñas. Además de la pobreza, las características familiares, las condiciones del contexto educativo y las circunstancias del entorno socio-político son factores que influyen en su aparición y mantenimiento (Holgado, Maya-Jariego, Palacio, \& Oviedo-Trespalacios, 2016).
Los contextos con elevada prevalencia de trabajo infantil se caracterizan por un bajo nivel socioeconómico, además de niveles elevados de analfabetismo y una escasa urbanización del entorno. La presencia en la familia de un clima favorable hacia la implicación de los niños en actividades laborales, junto con un mayor número de hijos, ser el primogénito en el hogar familiar, o una elevada tasa de violencia intrafamiliar, constituyen factores de riesgo significativos (Emerson \& Souza, 2008; Holgado et al., 2014). A ello se añade el fenómeno del desplazamiento del ámbito rural al ámbito urbano (Pinzón-Rondón, Briceño-Ayala, Botero, Cabrera, \& Rodríguez, 2006). Otros factores de riesgo son las restricciones para recibir créditos, la distancia a la escuela y una baja tasa de ocupación de las personas adultas responsables del hogar (Acevedo, Quejada, \& Yánez, 2011).

El trabajo infantil forma parte de un círculo vicioso vinculado a procesos económicos, sociales y culturales. En algunas comunidades peruanas, el trabajo que ejercen los menores es para los adultos una muestra de responsabilidad e independencia (Emerson \& Souza, 2003; Lieten $\&$ Strehl, 2015; Maya-Jariego, 2017). Algunas familias con hijos trabajadores asumen que se trata de una actividad positiva para su desarrollo. Ello disminuye la probabilidad de colaboración en programas de intervención psicoeducativa, lo que contribuye al mantenimiento del problema (Amar, Crisson, Palacio-Sañudo, Orozco, \& Pezzano, 2012).

También influyen las condiciones de los centros educativos. En contextos de riesgo, los colegios suelen presentar problemas de accesibilidad, de falta de formación de los docentes o de escasez de recursos (Romero et al., 2012). A ello se une el hecho de que las familias pueden sopesar el beneficio económico a corto plazo, frente a un contexto educativo deficiente, cuyos beneficios no están garantizados y ocurren a largo plazo (Emerson \& Souza, 2007). 


\section{Consecuencias del trabajo infantil en el desarrollo psicosocial}

\section{Autoestima}

Los niños y niñas que realizan actividades laborales presentan relaciones disfuncionales con su entorno cercano. Los menores trabajadores se ven sometidos, en ocasiones, a un proceso de estigmatización social por parte de los menores no trabajadores, lo cual repercute negativamente en su autoestima (de Baessa, 2008; Tabernero, Serrano, \& Mérida, 2017). Los menores trabajadores sufren un desarraigo temprano de los lazos familiares, debido al tiempo que pasan fuera del hogar, que afecta a la construcción de su autoestima (OIT, 2007). En las zonas rurales el trabajo se concibe como un contexto de aprendizaje más importante que la escuela, de forma que dedicarse a actividades laborales no correlaciona negativamente con la autoestima (Ponce, 2012). Sin embargo, en zonas urbanas, con el abandono del contexto educativo y la implicación en actividades laborales peligrosas, la autoestima puede verse afectada y coincidir con la aparición de conductas antisociales en los menores. En ambos escenarios, rurales y urbanos, la educación de calidad es la herramienta más importante para la construcción saludable de la autoestima del menor y la intervención con familias resulta clave para promover entornos seguros (Cabrera Cruz \& Díaz, 2011).

\section{Autoeficacia}

La autoeficacia se define como los juicios sobre las propias capacidades, que permiten al individuo establecer cursos de acción para conseguir sus objetivos (Bandura \& Walkers, 1974). En zonas rurales, donde el contexto educativo puede adolecer de falta de capacidades y recursos, los niños se auto-perciben como competentes en la actividad laboral concreta que realizan, aunque sus niveles de autoestima general sean bajos. La supervisión y el reforzamiento positivo de las familias en contextos rurales y la baja calidad de los recursos de las escuelas para la mejora de sus competencias, dificultan romper el ciclo del trabajo infantil. Sin embargo, en contextos urbanos, donde las escuelas son comparativamente más efectivas para mejorar las capacidades cognitivas, no se observa la misma intensidad en la relación entre trabajo infantil y autoeficacia (Ponce, 2012).

\section{Habilidades Sociales}

El trabajo infantil no solo afecta a cómo se sienten los menores con ellos mismos sino que implica también un déficit en el desarrollo de competencias para las relaciones con las personas de su entorno, a corto y largo plazo. La dedicación a actividades laborales influye en los resultados académicos lo que puede conllevar la aparición de malestar emocional y síntomas depresivos (Rosenberg, Schooler, \& Schoenbach, 1989). También repercute negativamente en las habilidades sociales y las actitudes prosociales (Amar et al., 2008). En los estudios comparativos, los menores trabajadores suelen mostrar una menor capacidad de autorregulación emocional, menos empatía y niveles más altos de ansiedad y estrés. La dificultad para sobrellevar situaciones conflictivas en su vida cotidiana se debe al escaso desarrollo de las habilidades sociales básicas, que se suelen aprender y desarrollar en los contextos educativos y de ocio (Palacio, Amar, Madariaga, Llinás, \& Contreras, 2007).

\section{Rendimiento académico y asistencia escolar}

El bajo rendimiento académico de los menores trabajadores se relaciona con el cansancio, que puede dificultar la atención y el esfuerzo necesarios para llevar a cabo las actividades y tareas en el centro educativo, y con la dedicación horaria, que puede restar tiempo al estudio y generar problemas para asistir al centro, aumentando el absentismo escolar (Guzmán, Guzmán, Ríos, \& Rivera, 2015; Post, 2011). La diversificación de los horarios escolares en países en vías de desarrollo puede facilitar el acceso 
al trabajo de los niños, debido a la posibilidad de compaginar la jornada escolar con la jornada laboral. La existencia de horarios escolares flexibles, de mañana, tarde o noche, puede contrarrestar la penalización de las prácticas laborales (Rodríguez \& Vargas, 2008). También ser el mayor de los hermanos, tener un mayor número de hermanos a cargo, ser niña y dedicarse al servicio doméstico, o acudir a una escuela con un idioma diferente de la lengua nativa, son factores con una influencia significativa en la asistencia a la escuela y aumentan la probabilidad de repetir curso (Patrinos \& Psacharopoulos, 1995).

\section{Impacto de las intervenciones psicoeducativas en la autoestima, autoeficacia, habilidades sociales y rendimiento académico}

En los centros educativos se incorporan programas extracurriculares para fortalecer su papel de espacios seguros de aprendizaje y dinamizar las relaciones entre las familias, los alumnos y la comunidad. Los profesores y los facilitadores actúan como modelos y guías de conducta y se consiguen mejoras en el uso del lenguaje socio-emocional, en las relaciones alumno-profesor y en el aprendizaje cooperativo (Villaseñor, 2018). En los programas extracurriculares los facilitadores trabajan con los menores en la mejora de competencias psicosociales de manera directa, mediante actividades dirigidas exclusivamente a los menores o de manera integrada con programas que abarcan a la familia, a los centros y a la comunidad.

En los programas dirigidos a menores se entrenan habilidades sociales de asertividad, empatía, negociación y escucha activa (Trianes, 1996). Las actividades para la mejora de las competencias psicosociales, como el teatro y el juego, transforman el autoconcepto de los menores y facilitan que reflexionen y adquieran conciencia sobre sus propias fortalezas y debilidades (Newman \& Dusenbury, 2015; Trianes \& García, 2002). Estas actividades influyen en las conductas antisociales de los menores, fomentan el desarrollo de competencias socioemocionales y mejoran los resultados académicos. A estos cambios se le suma un mejor comportamiento en el aula, una mayor capacidad para manejar el estrés y los problemas emocionales, así como juicios más positivos sobre ellos mismos, los demás y la escuela (Durlak, Weissberg, Dymnicki, Taylor, \& Schellinger, 2011).

La intervención en las competencias psicosociales de los menores es primordial para la obtención de buenos resultados académicos, para un buen desarrollo de la cognición y una adecuada adaptación al contexto escolar (Zins, Bloodworth, Weissberg, \& Walberg, 2004). La mejora del autoconocimiento y la autoestima permite valorar de manera más acertada la repercusión que tienen las propias acciones en los demás (Álvarez, Saldaña, Muñoz, \& Portela, 2009; Guo et al., 2015).

Las competencias psicosociales se trabajan de manera integral en los programas que incluyen la promoción del desarrollo socioemocional de los menores y el trabajo con la familia. Cuando se incluye a las familias en las intervenciones se obtienen resultados positivos en la reducción del aislamiento, en la impulsividad y en la asistencia escolar de los menores. Esta intervención conjunta permite que los padres y madres tomen conciencia de las necesidades de los hijos (Bartau \& de la Caba, (2009). Las competencias psicoeducativas tienen influencia en la autopercepción y en la autoestima cuando las familias interactúan con los cambios que se observan en los menores, amplían sus conocimientos sobre otras formas de educar y tienen la necesidad de compartir conocimientos y experiencias con la comunidad (Torres, Suárez, \& Rodrigo, 2014).

Los programas que trabajan con menores y con su contexto más cercano consiguen un mayor impacto en los participantes cuando se integran en el contexto educativo y comunitario, trabajando para fortalecer las competencias de los agentes implicados y facilitar la creación de coaliciones; y cuando contribuyen al desarrollo de las competencias psicosociales básicas en los 
menores, sentando las bases para el desarrollo de mejoras sostenibles a largo plazo. Los cambios psicosociales en los menores se establecen como objetivo primordial para la obtención de buenos resultados académicos, para un buen desarrollo de la cognición y una adecuada adaptación al contexto escolar (Zins et al., 2004). El impacto significativo en las habilidades sociales y en el rendimiento académico se manifiesta en el registro de puntuaciones más altas en comportamientos prosociales, en un mayor número de personas en la red social de los menores, en mayor autocontrol y en el manejo de conflictos en la escuela (Wortonet al., 2014).

Los programas extracurriculares están relacionados además con el desarrollo cotidiano del centro porque se ajustan a las características de la comunidad y a las necesidades de la población con la que se está interviniendo. Cuando el entorno comunitario es estable y está bien organizado, las familias tienden a relacionarse en torno al centro educativo y se implican en mayor medida en los programas de intervención. Esto fomenta la participación de los hijos en actividades positivas para su desarrollo psicosocial y, repercute en la reducción del trabajo infantil (Maya-Jariego, Holgado, Márquez, \& Santolaya, 2018). Por ello, la creación de alianzas entre agentes comunitarios, familias y responsables de los centros educativos es fundamental para el impacto significativo de las intervenciones en estos contextos (Mart, Dusenbury, \& Weissberg, 2011).

El esfuerzo de los facilitadores de los programas para la implicación de los menores, la familia, la comunidad y los centros educativos para conseguir resultados en el desarrollo psicosocial del menor (Márquez, Holgado, \& Maya-Jariego, 2019) tiene relación con la asistencia e implicación de los menores en la escuela, que es esencial para el éxito de las intervenciones (Romero, Amar, Palacio, Madariaga, \& Sierra, 2012). Esta mejora de la asistencia, se relaciona con el compromiso generado por los programas en los menores y sus familias (Quiroga, 2006). La evaluación conjunta de las actividades, permitirá que se identifican coincidencias en cuanto a los cambios observados (Peters et al., 2010).

\section{La experiencia de Edúcame Primero Perú}

En este estudio se presenta la evaluación del impacto del programa Edúcame Primero desarrollado en Lima (Perú). El programa fue diseñado para que los menores trabajadores (escolarizados o no), participaran en actividades de formación integral en competencias psicosociales y educativas en un espacio seguro de aprendizaje y ocio, alejado del contexto laboral.

El programa parte de un conjunto de experiencias piloto aplicadas en diferentes países de Centroamérica, principalmente en República Dominicana, de donde es originaria la estrategia principal del programa llamada Espacios Para Crecer. Tras la creación de una serie de alianzas entre diversas organizaciones internacionales, surgió el programa Edúcame Primero Colombia que se aplicó entre 2008 y 2010 en siete departamentos de Colombia, para la prevención del trabajo infantil, con la participación de más de 10.000 niños, como parte de una iniciativa de cooperación al desarrollo.

Tras la implementación en Colombia, se sistematizaron los resultados obtenidos en cuanto a la reducción del número de menores trabajadores y la mejora en los resultados académicos y de las competencias psicosociales, con el fin de facilitar su transferencia a Lima (Perú) entre 2015 y 2016 (Holgado et al., 2014). Ello permitió ajustar la estrategia al contexto peruano, con una implementación más breve, enfatizando el trabajo con las familias y teniendo en cuenta la cultura de trabajo que existe en el contexto peruano por la presencia de colectivos de menores trabajadores agrupados en movimientos nacionales que reivindican unos derechos básicos para su desarrollo (Márquez et al., 2019).

En este artículo exploramos el impacto del programa Edúcame Primero Perú en las competencias psicosociales de los menores participantes. Se analizan las consecuencias que la intervención tiene en la relación entre el centro educativo, las familias $y$ los menores, valorando el centro como espacio seguro y de referencia para las 
familias. Esto nos permitirá explorar como los programas extracurriculares conectan las dinámicas organizativas con dinámicas familiares o comunitarias para conseguir cambios favorables en los participantes.

\section{METODOLOGÍA}

\section{Participantes}

La evaluación del impacto se centró en 48 alumnos de entre 8 y 13 años $(M=10,38$, $\mathrm{DT}=1,22$ ), de los que se hizo seguimiento a lo largo de los 10 meses de implementación del programa. De ellos 18 eran niñas (el 37,5 \%) y 30 niños (el $62.5 \%$ ). Como se resume en la Figura 1, se trata de un subgrupo seleccionado de un colectivo de 151 estudiantes que inició su participación en los Espacios para Crecer en tres colegios de la periferia de Lima. Concretamente, se eligieron aquellos niños y niñas que, de acuerdo con la evaluación previa, se dedicaban a actividades laborales o estaban en alto riesgo de hacerlo. Los criterios de selección para los participantes en esta segunda fase de la intervención fueron (a) el tiempo y el grado de dedicación a actividades laborales remuneradas, (b) la intención declarada de trabajar y (c) el lugar de trabajo (fuera o dentro del hogar). En total, 36 alumnos fueron asignados al grupo de tratamiento, que recibió los talleres de "Espacios para Crecer”, y 12 al grupo de comparación.

\section{Figura 1.}

Evolución del número de menores participantes en Edúcame Primero Perú procedentes de los centros PS y MU, ubicados en Villa el Salvador (VES), y NS, situado en Jicamarca.

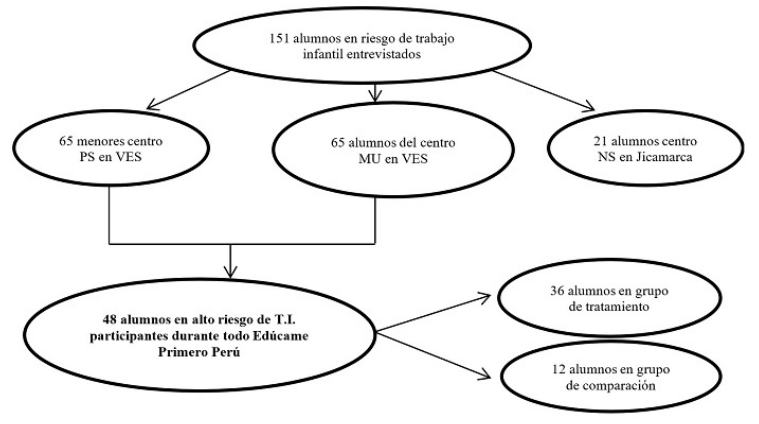

Procedimiento e instrumentos

Se utilizó un diseño de métodos mixtos. En los centros educativos se combinó (a) la observación participante y la recogida de información cualitativa con (b) la evaluación del impacto a través de indicadores cuantitativos antes y después de la implementación de la intervención.

Por lo que respecta al componente cualitativo, las facilitadoras del programa recogían y codificaban la información diaria de la intervención en cada centro en diarios semanales de campo, siguiendo el esquema de la Tabla 1. En los diarios se incluía el resumen de las entrevistas con las familias; el diario de las sesiones de los Espacios Para Crecer; las reuniones de seguimiento con los docentes y el equipo directivo; el contacto con los padres; las actividades de difusión del programa; el contacto con líderes comunitarios; la atención psicológica a familias y menores; las actividades de formación a las familias y los docentes; y otras actividades. Todo ello proporcionó una gran cantidad de información sobre el proceso de implementación del programa. Complementariamente, se realizaron entrevistas cualitativas semiestructuradas a menores durante las últimas semanas de intervención. 


\section{Tabla 1}

Clasificación de la información recogida durante la implementación de Edúcame Primero Perú

\begin{tabular}{|c|c|c|c|}
\hline Técnica & $\begin{array}{l}\text { Definición } \\
\end{array}$ & Informantes & Datos \\
\hline $\begin{array}{l}\text { Estudios de } \\
\text { casos }\end{array}$ & $\begin{array}{l}\text { Recopilación de información: } \\
\text { Responder cómo y por qué } \\
\text { (entrevista, observación y } \\
\text { documentación de cada alumno) }\end{array}$ & Menores & $\begin{array}{l}\text { Información especifica de } \\
\text { las circunstancias de cada } \\
\text { menor (pre-postest, } \\
\text { situación familiar, etc.) }\end{array}$ \\
\hline $\begin{array}{l}\text { Grupos } \\
\text { representativos }\end{array}$ & $\begin{array}{l}\text { Conversaciones con miembros de la } \\
\text { población beneficiaria }\end{array}$ & $\begin{array}{l}\text { Coordinadores, docentes y } \\
\text { representantes de los padres y } \\
\text { madres de los centros } \\
\text { educativos y de la comunidad }\end{array}$ & $\begin{array}{l}\text { Información sobre la } \\
\text { preparación comunitaria } \\
\text { de los contextos y el } \\
\text { impacto que el programa } \\
\text { ha tenido en la población }\end{array}$ \\
\hline Entrevistas & $\begin{array}{l}\text { Formales o informales con registro } \\
\text { de respuestas }\end{array}$ & $\begin{array}{l}\text { Menores participantes } \\
\text { Coordinadores de primaria }\end{array}$ & $\begin{array}{l}\text { Información sobre cómo } \\
\text { les ha parecido el } \\
\text { programa y lo cambios } \\
\text { percibidos }\end{array}$ \\
\hline Observación & $\begin{array}{l}\text { Registro diario (quien participa, qué } \\
\text { sucede, cuándo, dónde y cómo) }\end{array}$ & $\begin{array}{l}\text { Menores } \\
\text { Familias }\end{array}$ & $\begin{array}{l}\text { Datos de participación e } \\
\text { implicación de cada } \\
\text { participante en la sesión }\end{array}$ \\
\hline Cuestionarios & $\begin{array}{l}\text { Conjunto de preguntas de encuesta } \\
\text { codificables }\end{array}$ & & \\
\hline $\begin{array}{l}\text { Análisis de } \\
\text { documentos } \\
\text { escritos }\end{array}$ & Registros $\mathrm{y}$ bases de datos & $\begin{array}{l}\text { Obtenida en los centros de } \\
\text { cada menor. }\end{array}$ & Registro de calificaciones \\
\hline
\end{tabular}

Por su parte, la evaluación pretestpostest se basó en indicadores de habilidades sociales, autoeficacia, autoestima y rendimiento académico.

Habilidades sociales. Se aplicó la versión española de la escala Matson Evaluation of Social Skills with youngsters (MESSY). Los 5 factores seleccionados proceden del estudio de Ipiña, Molina \& Reyna (2011) de una evaluación con menores con edades comprendidas entre los 7 y 13 años. Los 62 ítems son los correspondientes a los utilizados en la adaptación española de la escala MESSY para niños de Trianes, Mena, Muñoz, García y Cardelle-Elawar (2002). Este instrumento permite evaluar habilidades sociales específicas que pueden ser clasificadas como conductas adaptativas o no adaptativas. Este instrumento está adaptado para niños, adolescentes y jóvenes de entre 4 y 18 años; y cuenta con 62 ítems que se evalúan en una escala tipo Likert $(1=$ Nunca, $2=$ a veces, $3=A$ menudo, $4=$ siempre). Las aplicaciones pre-test y post-test coincidieron en la obtención de un alfa de Cronbach de 0.84.

Auto-eficacia. Se utilizó la Escala de Autoeficacia para Niños (Bandura, 1990; Pastorelli, Caprara, Babaranelli, Rola, Rozsa \& Bandura, 2001). Esta escala evalúa la eficacia percibida del sujeto en los ámbitos social y académico, y cuenta con un factor para valorar la capacidad de autorregulación. Este instrumento constaba originalmente de 54 ítems (Bandura, 1990) y pasó a 35 en la adaptación española (Carrasco \& Del Barrio, 2002). Los ítems se evalúan con una escala de 1 a 5 puntos
$(1=$ fatal, $2=$ no muy bien, $3=$ bien; $4=$ muy bien; y $5=$ fenomenal). La versión utilizada en nuestro estudio fue ajustada al contexto de implementación del programa con 34 ítems, eliminándose el ítem original "del sexo opuesto" por considerarse redundante con respecto al ítem más general "Qué tal se te da hacer y mantener amigos". La escala obtuvo un indicador de fiabilidad de 0.92 en el pretest y de 0.89 en el postest, ambos muy cercanos al indicador de fiabilidad original.

Autoestima. En tercer lugar se utilizó la escala de Autoestima de Coopersmith según la adaptación para niños y adolescentes. La versión de Prewitt-Díaz (1984) consiste en 58 ítems que informan de la autoestima en cuatro ámbitos diferenciados: autoestima general, autoestima social, autoestima referida al hogar/padres, y escolar-académica. El coeficiente alfa de Cronbach fue de 0.70 en 2015 y 0.65 en 2016.

Rendimiento académico. Los participantes contestaron a cuatro preguntas para evaluar el rendimiento académico en el área de matemáticas. Estos cuatro ítems provienen del área lógico-matemática para alumnos de primaria de la Prueba CRECER 1998 para la evaluación nacional del rendimiento estudiantil en Perú (Unidad de Medición de la Calidad Educativa y Grupo de Análisis para el Desarrollo, 2001). Las dos primeras preguntas eran problemas sobre el sistema métrico decimal (aritmética) y las dos últimas sobre figuras geométricas y líneas paralelas (geometría). Complementariamente, se recogieron las calificaciones de los alumnos participantes en los Espacios Para Crecer del ciclo anterior al comienzo de la intervención (diciembre 2014) y en una fase intermedia de la implementación del programa (en diciembre 2015). Durante la intervención se realizó también un monitoreo semanal en el aula sobre la situación de los menores participantes en el programa. 
Análisis de datos

Para el análisis de resultados cuantitativos se ha utilizado el paquete estadístico SPSS 24. Debido a la distribución no paramétrica de los datos, se ha elegido la prueba Z de Wilcoxon para la comparación de medias de las variables consideradas (indicadores de autoestima, autoeficacia y habilidades sociales). Por otro lado, para el análisis de la información cualitativa se ha seguido una estrategia iterativa con comparación interjueces basada en la reducción de datos y la identificación y descripción de categorías de contenido vinculadas a la percepción de los cambios en los indicadores mencionados en niños, familias y docentes.

\section{RESULTADOS}

\section{Impacto psicosocial y rendimiento académico}

La evolución de aspectos psicosociales de los 48 menores participantes en el programa está resumida en la Tabla 2. En los participantes se observa un aumento significativo en la escala de autoestima $(Z=-3.04, p<0.01)$ y un descenso en la autoeficacia percibida $(Z=-3.92, p<$ $0.01)$ y en el auto-informe de habilidades sociales $(Z=-2.08, p<0.05)$. La mejora percibida en autoestima se da especialmente en el ámbito académico $(Z=-2.19, p<0.03)$. Por su parte, el descenso relativo en la autoeficacia percibida se da en los factores social $(Z=-3.08, p<0.01)$ y de autorregulación del propio comportamiento $(\mathrm{Z}=$ -2.99, $p<0.01)$, pero no en el ámbito académico. Finalmente, los participantes puntúan menos en la segunda observación en las competencias para las relaciones de amistad $(Z=-4.17, p<0.01)$ y los comportamientos de cortesía $(Z=-4.31, p<$ $0.01)$, a la vez que aumenta ligeramente el autoinforme de ansiedad social $(Z=-2.10, p<0.05)$.

Atendiendo a los dos grupos del programa, el descenso en el autoinforme de las habilidades sociales se observa en el grupo de tratamiento $(Z$ $=-2.19, p<=0.03)$ pero no en el grupo de comparación. Por lo que respecta a la autoestima, el grupo de tratamiento aumenta su valoración en el ámbito académico $(Z=-1.8, p<0.08)$.

Tabla 2

Puntuaciones de las escalas de autoestima, autoeficacia y habilidades sociales de los participantes en Edúcame Primero Perú $(N=48)$

\begin{tabular}{|c|c|c|c|c|c|}
\hline \multirow{2}{*}{ Escalas } & \multicolumn{2}{|c|}{2015} & \multicolumn{2}{|c|}{2016} & \multirow{2}{*}{$\begin{array}{c}\mathrm{Z} \\
\text { (Wilcoxon) }\end{array}$} \\
\hline & $\mathrm{M}$ & $\mathrm{DE}$ & $\mathrm{M}$ & $\mathrm{DE}$ & \\
\hline Autoestima & 0.63 & 0.09 & 0.66 & 0.10 & $-3.04 * *$ \\
\hline Autoeficacia & 3.97 & 0.59 & 3.55 & 0.47 & $-3.92 * *$ \\
\hline HHSS & 3.23 & 0.29 & 3.06 & 0.28 & $-2.08^{*}$ \\
\hline \multicolumn{6}{|l|}{ Factores de Autoestima } \\
\hline Autoestima general & 0.62 & 0.13 & 0.66 & 0.14 & $-2.09 *$ \\
\hline Autoestima social & 0.62 & 0.19 & 0.67 & 0.24 & -1.37 \\
\hline Autoestima escolar-académica & 0.71 & 0.16 & 0.78 & 0.18 & $-2.19^{*}$ \\
\hline Autoestima familiar & 0.63 & 0.17 & 0.67 & 0.25 & -0.7 \\
\hline Escala de Mentira & 0.57 & 0.15 & 0.51 & 0.16 & -1.75 \\
\hline \multicolumn{6}{|l|}{ Factores de Autoeficacia } \\
\hline Autoeficacia social & 3.90 & 0.69 & 3.55 & 0.48 & $-3.08^{* *}$ \\
\hline Autoeficacia Académica & 3.26 & 0.59 & 3.35 & 0.56 & -1.09 \\
\hline Autoeficacia Autorregulatoria & 4.54 & 0.76 & 4.14 & 0.59 & $-2.99 * *$ \\
\hline \multicolumn{6}{|l|}{ Factores de Habilidades Sociales } \\
\hline Agresividad/conducta antisocial & 3.38 & 0.38 & 3.35 & 0.40 & -0.02 \\
\hline HHSS apropiadas & 3.16 & 0.36 & 2.78 & 0.42 & $-4.31^{* *}$ \\
\hline Amistad & 3.12 & 0.52 & 1.38 & 0.30 & $-4.17 * *$ \\
\hline Exceso de confianza/celos/soberbia & 3.21 & 0.57 & 3.21 & 0.59 & -0.43 \\
\hline Soledad/ansiedad social & 2.85 & 0.69 & 3.1 & 0.69 & $-2.10^{*}$ \\
\hline
\end{tabular}

Para completar esta evaluación, en la tabla 3 hemos resumido las calificaciones académicas de los participantes en el programa así como sus resultados en las tareas de evaluación matemática. Las calificaciones académicas muestran, en general, estabilidad a lo largo del periodo observado. Solo se observa una puntuación ligeramente más baja en 2016 en la asignatura de personal-social $(\mathrm{Z}=-2.24, p$ $<0.05)$, que corresponde a los cambios en el grupo de tratamiento $(Z=2.11, p<0.05)$. Por lo que respecta a las tareas de evaluación matemática, obtuvimos una mejora en una tarea de aritmética $(Z=-2.18, p<0.03)$ y en una tarea de geometría $(Z=-2.29, p<0.02)$. También se dio una puntación peor en una segunda tarea de geometría $(Z=-4.00, p<0.01)$.

Todos estos cambios corresponden al grupo de tratamiento, que mejoran en el ejercicio sobre sistema decimal $(Z=-3.0, p=0.01)$ y en el ejercicio de figuras geométricas $(Z=-2.48, p<$ $0.02)$ y empeoran en la calificación de las líneas paralelas $(Z=6.12, p=0.01)$. 
Tabla 3

Calificaciones escolares y puntuaciones en las tareas de matemáticas de los participantes en Edúcame Primero Perú $(N=48)$

\begin{tabular}{lccccc}
\hline \multirow{2}{*}{\multicolumn{1}{c}{ Tareas }} & \multicolumn{2}{c}{$\begin{array}{c}\text { Calificaciones } \\
\text { previas a EPP }\end{array}$} & \multicolumn{2}{c}{$\begin{array}{c}\text { Calificaciones en } \\
\text { etapa final de EPP }\end{array}$} & \multirow{2}{*}{ Z } \\
\cline { 2 - 5 } & $\mathrm{M}$ & $\mathrm{DT}$ & $\mathrm{M}$ & $\mathrm{DT}$ & \\
& 2.95 & 0.59 & 2.94 & 0.53 & -0.70 \\
\hline Matemáticas & 2.90 & 0.54 & 2.98 & 0.56 & -0.90 \\
Comunicación & 3.12 & 0.33 & 3.10 & 0.37 & -1.00 \\
Arte & 3.19 & 0.40 & 3.06 & 0.32 & $-2.24^{*}$ \\
Personal-Social & 3.02 & 0.16 & 3.15 & 0.41 & $-2.24^{*}$ \\
Ed. Física & 3.05 & 0.32 & 3.06 & 0.33 & -1.00 \\
Religión & 3.15 & 0.42 & 3.10 & 0.37 & -0.58 \\
Ciencia y Ambiente & 3.06 & 0.27 & 3.06 & 0.32 & -0.34 \\
Media total asignaturas & 0.27 & 0.44 & 0.44 & 0.50 & $-2.18^{*}$ \\
Aritmética (metros) & 0.31 & 0.47 & 0.34 & 0.48 & -0.20 \\
Aritmética (litros) & 0.67 & 0.47 & 0.17 & 0.38 & $-4.00^{* *}$ \\
Geometría (figuras) & 0.37 & 0.49 & 0.59 & 0.50 & $-2.29^{*}$ \\
Geometría (rectas) & 0.41 & 0.25 & 0.29 & 0.23 & -0.11 \\
Media total tareas & & & & & \\
\hline
\end{tabular}

Nota. $* p<0.05, * * p<0.001$

Percepción de los cambios por parte de los estudiantes, los padres y los docentes

\section{Autoestima}

En los Espacios para Crecer, más de un tercio de las actividades estaban relacionadas con el autoconocimiento y la valoración del entorno cercano (concretamente, los módulos Yo soy especial y Mi familia, mi comunidad más cercana). A lo largo de la implementación se observó que los ejercicios para la valoración de la diversidad propiciaron una identificación más activa de las emociones y una valoración positiva de los sentimientos.

"Miss yo ya no me siento mal por ser mayor que mis compañeros, ahora nos llevamos mejor y salgo al recreo" [Alumna del centro Peruano-Suizo]

Los padres y profesores informaron de cambios positivos en la autopercepción de los niños participantes en el programa que llevaban a cambios iniciales en la confianza y en la autovaloración y en el comportamiento social, relacionados con la implicación en los Espacios para Crecer.

"Mi niña es muy reservada y se la pasa en la casa. Ahora está contenta porque acá no le da vergüenza hacer teatro o jugar con sus compañeros de salón" [Alumna del centro Max-Uhle]

\section{Autoeficacia}

Durante las escuelas de padres y madres y en los contactos diarios con docentes y coordinadores de centros, familiares y responsables de los menores se facilitaba información acerca de la importancia de que los niños y niñas aprendieran a resolver conflictos, plantearse metas futuras y forjar un plan de ejecución para cumplir sus expectativas. Los familiares, especialmente las madres, nos informaban de las inquietudes y/o preocupaciones que tenían los participantes con respecto al éxito en los estudios o en otras actividades que requiriesen esfuerzo, observándose actitudes nuevas relacionadas con la autoeficacia percibida.

"Miss, mi niña está preocupada porque no va poder ser psicóloga como tú porque para eso hay que saber mucha comunicación y a ella no se le da bien ese curso" [Padre del centro educativo Max-Uhle]

Estas observaciones coinciden con las respuestas que los alumnos del grupo de tratamiento dieron en las entrevistas, al finalizar la intervención. Algunos menores se atribuían bajas competencias para autorregular su conducta ante ciertas situaciones sociales que ocurrían en su familia y en su "salón".

"A mí me entra cólera muy rápido cuando me molestan y no sé cómo hacer para que se me pase" [Alumno del centro educativo Peruano- Suizo]

\section{Habilidades sociales}

El descenso en las puntuaciones del autoinforme de las habilidades sociales de cortesía, en la percepción de las relaciones de amistad y en la ansiedad social coincide con la identificación de emociones propias, relatadas por las familias y por los mismos alumnos durante las sesiones de los Espacios Para Crecer $\mathrm{y}$ en las actividades individuales y grupales, llevadas a cabo en los centros educativos. Los menores, coordinadores de centros y las familias informaron a las facilitadoras sobre cambios relevantes en el comportamiento y en las actitudes de los menores. Conforme avanzaba el 
programa, los participantes tomaban conciencia de la necesidad de mantener una comunicación asertiva y una escucha activa para la prevención de conflictos y malentendidos, tanto en el contexto familiar como en la escuela.

"Miss, yo quiero hablar con mi mamá pero no puedo porque no me escucha. Yo quiero aprender para poder contarle mis cosas sin que se moleste" [Alumna participante del centro Peruano-Suizo]

Las estrategias utilizadas por el programa, especialmente las sesiones de Espacios Para Crecer, permitían identificar emociones y conductas positivas para la comunicación con iguales y familias. Algunos de los testimonios de los menores informaban de ejemplos cotidianos de puesta en práctica de los ejercicios realizados en clase, en un proceso inicial de generalización del aprendizaje.

"Profesora, ya no pateo a mis compañeros porque luego me tienen miedo y así no puedo jugar y divertirme como hablamos el otro día en el taller" [Alumno participante del centro PeruanoSuizo]

\section{Rendimiento académico}

Los familiares de los menores veían en Edúcame Primero Perú un programa de apoyo extraescolar que permitía fortalecer los aprendizajes básicos del sistema educativo. Algunas docentes y madres comunicaban, durante los seguimientos semanales y en las reuniones planificadas, que estaban observando mejoras en la atención y concentración. Las actividades realizadas durante los talleres (forma en la que se conocía en los centros a la estrategia Espacios Para Crecer) a pesar de no tener, entre sus objetivos, contenidos académicos concretos, requerían el desarrollo de competencias como la atención selectiva, la memoria, la creatividad y la resolución de problemas. De esta manera, el trabajar estas competencias paralelamente a los contenidos centrales del programa (autoestima, autoeficacia y habilidades sociales) tenían un efecto en el área académica, lo que refleja el potencial de generalización del programa y de desarrollo integral de los niños participantes.

"Mi hija está menos distraída Miss, está interesada por unos temas muy raros pero sigue sacando buenas notas. Así que si aprende está bien" [Padre del centro educativo Peruano-Suizo]

Los responsables de aula y coordinadores indicaron cambios motivacionales en aquellos menores con mayor dificultad para participar activamente en actividades dentro y fuera del aula

"Este alumno ha subido en comunicación, antes nunca quería exponer sus trabajos y también lo veo más interesado en salir al recreo" [Docente del centro educativo Max-Uhle]

En general, se observa que los docentes, coordinadores de centros y familiares comunican cambios positivos en los menores referidos a la autovaloración, en la participación en actividades académicas y en la socialización dentro del contexto educativo. El programa es valorado como un espacio de aprendizaje y de respiro familiar, tanto para los menores como para sus padres o tutores. Además de ello, los menores manifestaban tomar conciencia de sus necesidades de autorregulación de los comportamientos negativos relacionados con las actividades con iguales dentro del contexto escolar e indicaban necesitar una mayor comunicación con sus padres. Por último, los menores mostraron interés por desarrollar competencias a largo plazo que le permitan alcanzar metas referidas a la formación superior. En cuanto a la relación con la familia, los niños indicaban interés por la mejora de la comunicación familiar.

Cambios personales en el contexto del trabajo infantil

Edúcame Primero Perú funciona como un catalizador del cambio personal y comunitario para la prevención del trabajo infantil. Para ello se centra en el desarrollo aspectos psicológicos y emocionales de los menores y promueve la 
concienciación de la población sobre la meta última de disminución del riesgo de trabajo infantil. Para completar la evaluación, resumimos primero la reacción hacia los talleres por parte de los participantes y sus familias; y, en segundo lugar, presentamos el impacto percibido en términos de prevención del trabajo infantil.

\section{Satisfacción general con los Espacios Para Crecer}

Los Espacios Para Crecer fueron la estrategia principal del programa, donde los niños participaban en actividades relacionadas con el desarrollo de conductas psicosociales positivas como la empatía, el cuidado de la familia y las personas queridas, el respeto por la naturaleza, el compromiso con el barrio y con el entorno del que se sienten parte, entre otras competencias. Los alumnos tenían un espacio seguro donde disfrutar, reír y aprender de una forma diferente. Esto unido a la justa distribución de los horarios de los talleres permitía la satisfacción generalizada de los participantes por la continuación del programa en el centro.

Por un lado, los alumnos participantes manifestaban su interés por continuar en los Espacios para Crecer por identificarlo con un espacio de distensión de sus tareas cotidianas.

"Miss yo no quiero que termine el taller porque aquí aprendemos con juegos y me río bastante" [Alumna centro Max-Uhle]

Además, los participantes manifestaban aprender tareas útiles e interesantes y tener deseo de extender las actividades a otros espacios.

"Me gustaría hacer ejercicios y juegos como los de taller con todo mi salón porque aprendemos cosas nuevas y así todos nos llevamos mejor" [Alumna centro Peruano-Suizo]

Por otro lado, las madres y familiares responsables de los menores participantes indicaban su satisfacción con lo que hacían sus hijos en los talleres y con otras acciones externas como la atención personalizada en casos concretos.
"Profesora mi hijo menor está muy cambiado desde que viene acá con usted, yo quisiera que viniera también otro hijo mayor que tengo que está un poco perdido" [Abuela del centro Peruano-Suizo]

Por último, los docentes y coordinadoras de los centros reconocían el impacto del programa y de su integración en la dinámica escolar.

"Señorita los alumnos han mejorado bastante, el alumno $X$, por ejemplo, no tenía control ante de que ustedes llegaran ahora todas las mamás hablan muy bien de los talleres y quieren apuntarse" [Coordinadora del centro PS].

Concepción del trabajo infantil, perspectivas de futuro y organización del tiempo de los menores

Edúcame Primero Perú trataba de fomentar el interés por la continuidad de los estudios y priorizar que los menores pasaran tiempo de ocio y de estudio dentro del centro educativo considerado un contexto seguro para el desarrollo psicosocial de los participantes. Los padres y tutores estaban motivados con las actividades que se hacían dentro del centro y reconocían la importancia de reducir el tiempo que los menores pasaban en entornos laborales. En este contexto el programa funciona como un espacio de respiro familiar, sobre todo al plantearse en la jornada contraria a la escolar. Esto alejaba, en algunos casos, a los niños y niñas del ambiente laboral de los padres, que no tenían que llevarlos consigo a los espacios de trabajo (aunque los menores no ejercieran tareas laborales en estos contextos).

"Yo no puedo controlar mucho a mis hijitos porque me la paso trabajando en el mercado por eso me gusta que vengan ustedes a hacer las tareas y también las llevo a danza en el colegio" [Madre del centro Max-Uhle].

En los barrios más pobres de Lima existe la idea de que el trabajo del menor es una ayuda y un apoyo a la familia y es habitual que los padres y madres cuenten con los hijos (sobre todo los de mayor edad) para el cuidado de hijos menores, la realización de tareas del hogar y la contribución en negocios familiares. Sin embargo, gracias a los contextos educativos y a las campañas nacionales 
de educación, las familias y los propios menores empiezan a estar más concienciados acerca del futuro. Durante la implementación del programa, algunos familiares informaron de su confianza en los centros educativos y reconocieron la importancia de la educación para el desarrollo y el futuro profesional de sus hijos e hijas. Edúcame Primero Perú se integró en la dinámica escolar y parte como una oferta dentro del centro educativo y facilitó espacios donde las familias y los menores pueden trasmitir sus deseos.

"Yo quiero que mi hija sea una profesional porque ese era mi sueño cuando yo era chiquita, yo sé que le exijo mucho para que ayude en la casa con sus hermanitas pero yo este año la voy a apoyar más para que se motive con el estudio" [madre de MaxUhle en una de las últimas escuelas de madres y padres]

La concepción de los padres respecto al trabajo en sus hijos e hijas tiene que ver, en muchas ocasiones, con la idea de que es un contexto de aprendizaje, incluso más útil que la escuela (sobre todo cuando se percibe que ésta no cumple con estándares mínimos de calidad). Pero la implementación de estrategias para promover los contextos educativos como espacios seguros (como son los Espacios Para Crecer), fomentan la apertura en la concepción de los padres sobre el estudio, dando prioridad al cumplimiento de las obligaciones durante la jornada escolar.

"Yo tengo que ayudar a mi papá los fines de semana porque él es constructor y quiere que lo acompañe pero durante la semana no me deja trabajar porque dice que tengo que sacar buenas notas" [alumno participante del centro Peruano Suizo]

Los menores participantes, durante el monitoreo continuado en los centros, en las sesiones de los Espacios para Crecer y en las entrevistas individualizadas al finalizar la intervención, indicaban su interés por obtener buenas calificaciones a pesar de tener que combinarlas con tareas del hogar.

"Miss, yo no puedo venir a los talleres porque mis padres trabajan de lunes a domingo y yo cuido a mis hermanitos, yo hago todas mis tareas en el recreo para avanzar" [alumno participante del centro MU]

Entre los niños y niñas que participaron durante toda la implementación, algunos referían a las facilitadoras su interés por conocer las posibles líneas de formación tras finalizar sus estudios secundarios.

"Miss mis papas me dicen que tengo que continuar estudiando pero yo no sé cómo se pueden conseguir becas para estudiar. Aquí solo conocemos la Universidad César Vallejo porque vino a explicarnos cosas pero está difícil la postulación” '[Alumna del centro MU]

\section{DISCUSIÓN}

Este estudio analizó el impacto de una intervención psicoeducativa en la prevención del trabajo infantil, documentó la toma de conciencia de los cambios comportamentales por parte de los participantes, y mostró la repercusión indirecta en el contexto familiar y educativo. El programa Edúcame Primero Perú se mostró efectivo de manera incipiente en la capacitación de los menores, la sensibilización de la población y la preparación de la comunidad para el cambio (Holgado, Santolaya, Maya-Jariego, Cueto, \& Anaya, 2015). Pese a que el impacto observado fue parcial en el periodo de implementación analizado, parece incidir en las concepciones sobre el trabajo infantil, que a su vez repercuten indirectamente en las competencias psicosociales y académicas de los menores. Los colegios se revelaron como centros de referencia para la comunidad, donde se construyen los cambios observados en los participantes, que alcanzan indirectamente al resto de la población (Márquez et al., 2019).

La incorporación del programa en los centros educativos logró un impacto en los participantes en tres niveles. En primer lugar en la autoestima, que aumentó de manera general en los participantes. Este cambio puede relacionarse con las actividades psicoeducativas que se llevaron a cabo con los familiares, centradas en la identificación de emociones, y dirigidas a promover la socialización y el juego en la escuela, 
en un entorno seguro (Cabrera Cruz \& Díaz, 2011). En segundo lugar, incidió en la reacción de los participantes al programa, que modificaron el juicio sobre sus propias capacidades (Bandura \& Walkers, 1974), y sobre las competencias que necesitan para sentirse más eficaces social y académicamente en un contexto urbano. El colegio refuerza el valor de la formación en el desarrollo personal, frente a la incorporación temprana al mundo laboral (Ponce, 2012). En tercer lugar, mejoró la percepción de las propias competencias, tanto las relacionadas con el entorno académico como las habilidades sociales (concretamente, la autoeficacia social y de autoregulación, y las competencias para la amistad y la cortesía).

El análisis del impacto trasciende la evaluación académica, pues se centró en aspectos socio-afectivos (Trianes, 1996), examinando los cambios en las competencias sociales y en el rendimiento académico de los participantes, junto con el ajuste comunitario de la intervención (Márquez et al., 2019). Comprobamos que los menores se convierten en protagonistas activos de su cambio personal y transfieren sus aprendizajes al hogar y a su relación con los iguales. Concretamente, se observaron más conductas cooperativas, las familias mencionaron su satisfacción con la intervención y destacaron el aumento en la motivación de sus hijos e hijas hacia el contexto educativo. Estos factores son claves para la protección frente a la depresión y se relacionan con dar valor a la repercusión que las propias acciones tienen en los demás (Guo et al., 2015).

Además de potenciar las relaciones positivas dentro y fuera del colegio, la intervención incidió en los antecedentes del trabajo infantil. Se pusieron en marcha estrategias para prevenir que los menores perdieran el interés en la formación y se promovieron competencias que facilitan la auto-regulación. Se trata de habilidades cognitivas que permiten el desarrollo de expectativas realistas con relación a sí mismo, reconociendo metas alcanzables y proyectándose con mayor facilidad hacia un futuro productivo, con mayor conciencia de las propias capacidades (Álvarez et al., 2009). Estas competencias frenan el ciclo que se inicia al asumir responsabilidades familiares (por ejemplo, trabajando en el hogar), y continúa al alejarse progresivamente del entorno académico, acercándose al entorno laboral.

Los menores mejoraron en el reconocimiento de sus propias capacidades y en el manejo de información sobre la propia cognición, o meta-cognición, lo cual resulta clave para una salud mental positiva (Marino et al., 2016). La creencia en sí mismo mejoró especialmente en términos de autoeficacia académica. Sin embargo, posiblemente tomaron conciencia de que necesitaban mejorar sus competencias sociales y la auto-regulación en contextos de interacción cotidianos. La influencia del contexto cercano, la constancia en las relaciones positivas y el establecimiento de unas perspectivas de futuro alcanzables son los factores que relacionan la autoeficacia con el rendimiento académico, por lo que es clave la toma de conciencia de las familias (Lacunza \& Cotinini de González, 2011). Los padres y madres de los participantes en el programa tomaron conciencia de la importancia de la educación, la comunicación o el desarrollo de competencias sociales en los menores. En su conjunto, se consiguió que los menores y su contexto se posicionaran en las etapas iniciales de preparación para el cambio en lo referente a la concepción del trabajo infantil.

Sin embargo, a lo largo del programa se observó un descenso en las habilidades sociales auto-informadas. Las habilidades sociales tienen un efecto indirecto en las competencias académicas (Jennings \& Diprete, 2010), contribuyen al desarrollo sociocognitivo y son el mejor predictor infantil de la adaptación y el desarrollo en la etapa adulta, por encima incluso del rendimiento académico (Hartup, 1992). Estas competencias se desarrollan de manera natural a través de la participación en grupos, ya sea con la familia, la escuela o los compañeros (Lacunza \& Contini de González, 2011), aunque en los contextos de riesgo puede asociarse con el despliegue de conductas disfuncionales. Otros indicadores mostraron una evolución positiva. Se valoró positivamente la organización de espacios alternativos a la jornada escolar. Las familias 
lo vieron como una oportunidad para trabajar competencias psicoeducativas y encontraron en los talleres un recurso que facilitaba el mantenimiento de una comunicación fluida en la escuela. Por lo demás, si los niños y niñas comparten espacios de comunicación y aprendizaje a través del juego, se sienten más satisfechos con su vida y hacen una autovaloración más positiva (Tonon, Laurito, \& Benatuil, 2018)

Los objetivos del programa se centraban en menores en situación de riesgo, con acciones dirigidas preferentemente a la prevención del trabajo infantil. No obstante, las diferentes partes interesadas reconocieron contribuciones del programa que pueden ser de utilidad en implementaciones futuras, especialmente para incorporar estrategias de ajuste comunitario (Aarons, Wells, Zagursky, Fettes, \& Palinkas, 2009). Para las facilitadoras el programa tiene un impacto en la prevención y la reducción del trabajo infantil, en la mejora de la situación de los menores en su comunidad, en la comunicación con su entorno y en la toma de conciencia sobre sus habilidades y competencias sociales. Por su parte, las familias reconocen el valor de las actividades extraescolares que aporta el programa y ven en la intervención una oportunidad para recibir orientación psicológica profesional y tener acceso a servicios psicosociales que no están disponibles durante la jornada escolar. Edúcame Primero Perú es percibido como un recurso para mediar ante conflictos familiares internos, conflictos individuales y dificultades en el desarrollo de los menores. En su opinión, también facilita la adaptación de los menores al centro educativo. Finalmente, para las coordinadoras de los colegios (directores o jefes de estudio), el programa es un recurso valioso y gratuito que permite la mediación entre la familia y la escuela, facilita la relaciones humanas y fomenta el intercambio de impresiones sobre la evolución de los alumnos con dificultades familiares y /o psicosociales.

\section{CONCLUSIONES}

En este estudio valoramos la evolución de un programa de prevención del trabajo infantil durante un corto periodo de aplicación. Esta intervención psicoeducativa mostró un impacto positivo en la mejora de la autoestima de los participantes, en algunos indicadores de rendimiento académico y en la implicación en actividades escolares. Sin embargo, durante el periodo analizado se observó un descenso en la auto-evaluación de las habilidades sociales y en la auto-eficacia percibida en el ámbito de la interacción social. Los participantes parecen haber tomado conciencia sobre sus capacidades personales y también sobre las carencias que tienen para relacionarse de manera positiva con su entorno, ya sea en el contexto familiar, académico o en las relaciones con compañeros. Posiblemente esto está reflejando el inicio de un cambio comportamental que requiere más tiempo para consolidarse y mostrarse de manera definida.

Sin embargo, pese a que el impacto psicosocial observado es reducido, el programa fue muy bien valorado por las familias y por los responsables de las escuelas. En general fue considerado un catalizador del cambio comportamental positivo:

"Mi hija siempre está nerviosa durante el almuerzo porque no quiere llegar tarde a los talleres. La veo más tranquila, ya no pelea tanto con sus hermanitos porque está ilusionada" [Madre del centro Educativo Peruano-Suizo]

Las diferentes partes involucradas en la intervención encontraron en los talleres una oportunidad para acceder a servicios psicológicos profesionales y un recurso para la mediación entre las familias y la escuela. Esto resulta especialmente significativo si tenemos en cuenta que la observación participante puso de manifiesto que la conexión de las familias con la escuela resultó clave en la implementación del programa y en el aumento progresivo de la asistencia a las actividades extraescolares por parte de los menores en mayor riesgo de trabajo infantil. 


\section{REFERENCIAS}

Aarons, G. A., Wells, R. S., Zagursky, K., Fettes, D. L., \& Palinkas, L. A. (2009). Implementing evidence-based practice in community mental health agencies: A multiple stakeholder analysis. American Journal of Public Health, 99(11), 2087-2095. https://doi.org/10.2105/AJPH. 2009.161711

Acevedo, K., Quejada, R., \& Yánez, M. (2011). Estudio transversal de los determinantes del trabajo infantil en Cartagena, año 2007. Revista Latinoamericana de Ciencias Sociales, Niñez y Juventud, 9(2), 589-606.

Álvarez, L. Y., Saldaña, C., Muñoz, J. A., \& Portela, V., (2009) Promoción de comportamientos pro-sociales y fortalecimiento de habilidades cognitivas, emocionales y conductuales en niños, niñas y adolescentes en situación de y en calle, de la ciudad de Bucaramanga. Poiésis, 9(18), 1-21. https://doi.org/10.21501/16920945.1 36

Amar, J., Palacio, J., Llinás, H., Puerta, L., Sierra, E., Pérez, A. M., \& Velásquez, B. (2008). Calidad de vida y salud mental positiva en menores trabajadores de Toluviejo. Suma Psicológica, 15(2), 385-404.

Amar, J. A., Crisson, E. S., Sañudo, J. P., Orozco, C. M., \& Pezzano, G. (2012). Trabajo infantil: Factores de riesgo y protección en familias del caribe colombiano. Barranquilla: Editorial Universidad del Norte.

Bandura, A. (1990). Perceived self-efficacy in the exercise of personal agency. Journal of Applied Sport Psychology, 2(2), 128-163. htt ps://doi.org/10.1080/10413209008406426

Bandura, A., \& Walkers, W. (1974). Aprendizaje social y desarrollo de la personalidad. Madrid: Alianza.

Bartau, I., \& de la Caba, M. A., (2009). Una experiencia de mejora de las habilidades para la parentalidad y el desarrollo sociopersonal de los menores en contextos de desprotección social. Psychosocial Intervention, 18(2), 135-151. ht tps://doi.org/10.5093/in2009v18n2a5
Cabrera Cruz, M. M., \& Díaz, M. N. (2011). Una mirada al trabajo infantil rural en Yucul San Ramón desde la percepción de niños niñas padres y madres de familia docentes y representantes de OSC. (Doctoral dissertation). Universidad Centroamericana, Nicaragua.

de Baessa, Y. (2008). Child labor in guatemalan children: an international pilot study of the emotional effects and consequences. Journal of Emotional Abuse, 8(3), 325-333. https://doi.org/10.1080/109 26790802313359

Durlak, J. A., Weissberg, R. P., Dymnicki, A. B., Taylor, R. D., \& Schellinger, K. B. (2011). The impact of enhancing students' social and emotional learning: A meta-analysis of school-based universal interventions. Child Development, 82(1), 405-432. https://doi.o $\mathrm{rg} / 10.1111 / \mathrm{j} .1467-8624.2010 .01564 . x$

Emerson, P. M., \& Souza, A. P. (2003). Is there a child labor trap? Intergenerational persistence of child labor in Brazil. Economic Development and Cultural Change, 51(2), 375-398. https://doi.org/10.1086/346003

Emerson, P. M., \& Souza, A. P. (2007). Child labor, school attendance, and intrahousehold gender bias in Brazil. The World Bank Economic Review, 21(2), 301-316. https://doi.org/10.1093/wber/lhm 001

Emerson, P.M., \& Souza, A. P. (2008). Birth order, child labor, and school attendance in Brazil. World Development, 36(9), 1647-1664. http s://doi.org/10.1016/j.worlddev.2007.09.004

Guo, S., Wu, Q., Smokowski, P. R., Bacallao, M., Evans, C. B., \& Cotter, K. L. (2015). A longitudinal evaluation of the positive action program in a low-income, racially diverse, rural county: Effects on selfesteem, school hassles, aggression, and internalizing symptoms. Journal of Youth and Adolescence, 44(12), 2337-2358. https://doi .org/10.1007/s10964-015-0358-1

Guzmán, M. F. S., Guzmán, M. N. S., Ríos, M. D. M. M., \& Rivera, M. M. (2015). Impacto de trabajo infantil en el rendimiento escolar de adolescentes en poblaciones 
rurales: estudio de caso. Revista Lasallista de Investigación, 12(1), 147-153. https://doi.or g/10.22507/rli.v12n1a13

Hartup, W. W. (1992). Friendships and their developmental significance. In H. McGurk (Ed.), Childhood social development: Contemporary perspectives ( $\mathrm{p}$. 175-205). Lawrence Erlbaum Associates, Inc.

Holgado, D., Maya-Jariego, I., Ramos, I., Palacio, J., Oviedo-Trespalacios, O., RomeroMendoza, V., \& Amar, J. (2014). Impact of child labor on academic performance: Evidence from the program "Edúcame Primero Colombia". International Journal of Educational Development, 34, 58-66. https:// doi.org/10.1016/j.ijedudev.2012.08.004

Holgado, D., Santolaya, F. J., Maya-Jariego, I., Cueto, R. M., \& Anaya, R. H. (2015). Preparación comunitaria y organizativa contra el trabajo infantil en tres colegios de barrios periféricos de Lima (Perú). Apuntes de Psicología, 33(3), 103-116. https://idus.u s.es/xmlui/handle/11441/64134

Holgado, D., Maya-Jariego, I., Palacio, J., \& Oviedo-Trespalacios, Ó. (2016). Two Profiles of Child Labor in the Colombian Caribbean Coast: Children Relocated to Suburban Areas Compared to the Key Role of Social and Labor Characteristics of Mothers in Urban Settings. In GTonon (Ed.), Indicators of quality of life in Latin America (pp. 251-273). Cham: Springer. https://doi.org/10.1007/978-3-31 9-28842-0_11

Ipiña, M. J., Molina, L., \& Reyna, C. (2011). Propiedades psicométricas de la Escala MESSY (versión autoinforme) en niños argentinos. Revista de Psicología (PUCP), 29(2), 245-264. http://revistas.pucp.edu.pe /index.php/psicologia/article/view/2468

Jennings, J. L., \& DiPrete, T. A. (2010). Teacher effects on social and behavioral skills in early elementary school. Sociology of Education, 83(2), 135-159. https://doi.org/1 $0.1177 / 0038040710368011$

Lacunza, A. B., \& Contini de González, N. (2011). Las habilidades sociales en niños y adolescentes. Su importancia en la prevención de trastornos psicopatológicos. Fundamentos en humanidades, 12(23), 159-182. https://www.redalyc.org/articulo. oa? id $=18424417009$

Lieten, G. K. \& Strehl, T. (2015) Child Street Life: an inside view of hazards and expectations of street children in Peru. New York, NY: Springer. https://doi.org/10.1007/978-3-31 9-11722-5

Marino, C., Vieno, A., Lenzi, M., Fernie, B. A., Nikčević, A. V., \& Spada, M. M. (2016). Personality traits and metacognitions as predictors of positive mental health in college students. Journal of Happiness Studies, 19(2) 1-15. https://doi.org/10.1007 /s10902-016-9825-y

Márquez, E., Holgado, D. \& Maya-Jariego, I. (2019). Innovation, dosage and responsiveness in the implementation of the program "Edúcame Primero Perú" for reducing child labor. Applied Research in Quality of Life, 14(3), 617-636. https://doi. org/10.1007/s11482-018-9608-1

Mart, A., Dusenbury, L., \& Weissberg, R. P. (2011). Social, emotional, and academic learning: Complementary goals for school-family partnerships. In S. Redding, M. Murphy \& Sheley P. (Eds.), Handbook on Family and Community Engagement(pp. 37-44). Lincoln, IL: Academic Development Institute / Center on Innovation \& Improvement.

Maya-Jariego, I. (2017). "But we want to work": The movement of child workers in Peru and the actions for reducing child labor. American Journal of Community Psychology, 60(3-4), 430-438. https://doi.org/10.1002/a jср. 12180

Maya-Jariego, I., Holgado, D., Márquez, E., \& Santolaya, F. J. (2018). The community role of schools in Jicamarca and Villa El Salvador (Peru): crosscutting behavior settings in personal networks. Psychosocial Intervention, 27(1), 1-11. https://doi.org/10. 5093/pi2018a3

Newman, J., \& Dusenbury, L. (2015). Social and Emotional Learning (SEL): A Framework 
for Academic, Social, and Emotional Success. In K. Bosworth (Ed.), Prevention Science in School Settings (pp. 287-306). New York, NY: Springer.

OIT. (2007). Trabajo infantil: causa y efecto de la perpetuación de la pobreza. San José: Programa Internacional para la Erradicación del Trabajo Infantil de la Oficina Internacional del Trabajo (IPEC).

OIT. (2004). Trabajo infantil. Un manual para estudiantes. Ginebra: Organización Internacional del trabajo.

OIT. (2017a). Ending child labour by 2025: A review of policies and programmes. World Report on Child Labour 2017 Geneva: International Labour Office.

OIT. (2017b). Results and trends, 2012-2016. Global estimates of Child Labor. Génova.: International Labour Office.

Palacio, J., Amar, J., Madariaga, C., Llinás, H., \& Contreras, K. (2007). La calidad de vida en salud en los menores trabajadores de Toluviejo (Sucre). Investigación $\mathbb{E}$ Desarrollo, 15(2), 366-393. https://www.red alyc.org/articulo.oa?id=26815206

Pastorelli, C., Caprara, G. V., Barbaranelli, C., Rola, J., Rozsa, S., \& Bandura, A. (2001). The structure of children's perceived selfefficacy: A cross-national study. European Journal of Psychological Assessment, 17(2), 87-97. https://doi.org/10.1027//1015-5759. 17.2.87

Patrinos, H. A., \& Psacharopoulos, G. (1995). Educational performance and child labor in Paraguay. International Journal of Educational Development, 15(1), 47-60. http s://doi.org/10.1016/0738-0593(94)E0001-5

Patrinos, H. A., \& Psacharopoulos, G. (1995). Family size, schooling and child labor in Peru. An empirical analysis. Journal of Population Economics, 10 (4), 387-405. https ://doi.org/10.1007/s001480050050

Peters, R. D., Bradshaw, A. J., Petrunka, K., Nelson, G., Herry, Y., Craig, W., \& Rossiter, M. (2010). The "Better Beginnings, Better Futures" ecological, community-based early childhood prevention project: Findings from Grade 3 to Grade 9. Monographs of the Society for Research in Child Development, 75(3), 1-176. https://doi.org/10.1111/j.154 0-5834.2010.00577.x

Pinzón-Rondón, A. M., Briceño-Ayala, L., Botero, J. C., Cabrera, P., \& Rodríguez, M. N. (2006). Trabajo infantil ambulante en las capitales latinoamericanas. Salud Pública de México, 48(5), 363-372. https://doi.org/10. 1590/S0036-36342006000500002

Ponce, C. (2012). Efectos heterogéneos del trabajo infantil en la adquisición de habilidades cognitivas. Avances de Investigación. Pobreza y Equidad. Lima: Grupo de Análisis para el Desarrollo (GRADE).

Post, D. (2011). Primary school student employment and academic achievement in Chile, Colombia, Ecuador and Peru. International Labour Review, 150(3-4), 255-278. https://doi.org/10.1111/j.1564-91 3X.2011.00116.x

Prewitt Diaz, J. O. (1984). A crosscultural study of the reliability of the Coopersmith Self Esteem Inventory. Educational and Psychological Measurement, 44(3), 575-581. https://doi.org/10.1177/00 13164484443005

Quiroga, B. M. (2006). Trabajo infantil en los niños y jóvenes beneficiarios del programa familias en acción: Una evaluación de impacto. Archivos de Economía 003650, Departamento Nacional de Planeación de España.

Rodríguez, J., \& Vargas, S. (2008). Escolaridad y trabajo infantil: patrones y determinantes de la asignación del tiempo de niños y adolescentes en Lima Metropolitana. Lima: Documento de trabajo 265 de la Pontificia Universidad Católica del Perú (PUCP)

Romero, V., Amar, J. J., Palacio, J., Madariaga, C., Sierra, E., \& Quintero, S. M. (2012). Factores familiares y sociales de alto riesgo asociados al trabajo infantil en ciudades de la Costa Caribe colombiana. Universitas Psychologica, 11 (2), 481-496. https://doi.org /10.11144/Javeriana.upsy11-2.ffsa

Rosenberg, M., Schooler, C., \& Schoenbach, C. (1989). Self-esteem and adolescent problems: Modeling reciprocal effects. 
American Sociological Review, 54(6), 1004-1018. https://doi.org/10.2307/209572 0

Tabernero, C., Serrano, A., \& Merida, R. (2017). Estudio comparativo de la autoestima en escolares de diferente nivel socioeconómico. Psicología Educativa, 23(1), 9-17. https://doi.org/10.1016/j.pse.2 017.02.001

Tonon, G., Laurito, M. J., \& Benatuil, D. (2019). Leisure, Free Time and Well-Being of 10 Years Old Children Living in Buenos Aires Province, Argentina. Applied Research in Quality of Life, 14, 1-22. https://doi.org/10. 1007/s11482-018-9612-5

Torres, A., Suárez, A., \& Rodrigo, M. J. (2014). Educar en Positivo: Primeros resultados y retos de futuro. Revista Iberoamericana de Sistemas, Cibernética e Informática, 11(2), 1-13. http://www.iiisci.org/journal/CV $\$ /$ ris ci/pdfs/AI001AI14.pdf

Trianes, M. (1996). iSe pueden conseguir unas relaciones interpersonales de calidad dentro del aula? Breve historia de una línea de trabajo. Cultura y Educación, 8(3), 37-48. https://doi.org/10.1174/1135640967 63270316

Trianes. M. V., \& García, A. (2002). Educación socio-afectiva y prevención de conflictos interpersonales en los centros escolares. Revista Interuniversitaria de Formación del profesorado, (44), 175-189. https://www.red alyc.org/pdf/274/27404409.pdf

Trianes, M. V., Mena, M. J. B., Muñoz, A., García, B., Cardelle-Elawar, M., \& Infante, L. (2002). Relaciones entre evaluadores de la competencia social en preadolescentes: Profesores, iguales y autoinformes. Anales de Psicología, 18(2), 197-214. https://www.redalyc.org/service/r 2020/downloadPdf/167/16718201/1

Unidad de Medición de la Calidad Educativa y Grupo de Análisis para el Desarrollo (2001). Análisis de ítems de las pruebas CRECER 1998. Lima (Perú): Ministerio de Educación.

Villaseñor, E.L. (2018). Reformas educativas. Su impacto en la innovación curricular y la formación docente. México D.F.: Ediciones Mínimas.

Worton, S. K., Caplan, R., Nelson, G., Pancer, S. M., Loomis, C., Peters, R. D., \& Hayward, K. (2014). Better Beginnings, Better Futures: Theory, research, and knowledge transfer of a community-based initiative for children and families. Psychosocial Intervention, 23(2), 135-143. https://doi.org /10.1016/j.psi.2014.02.001

Zins, J. E., Bloodworth, M. R., Weissberg, R. P., \& Walberg, H. J. (2007). The scientific base linking social and emotional learning to school success. Journal of Educational and Psychological Consultation 17(2-3), 191-210. https://doi.org/10.1080/1 0474410701413145

\section{Notas}

* Artículo de investigación. 